\title{
Energy Efficient Clustering Based Network Protocol Stack for 3D Airborne Monitoring System
}

\author{
Abhishek Joshi, Sarang Dhongdi, Rishabh Sethunathan, Pritish Nahar, and K. R. Anupama \\ BITS Pilani, K K Birla Goa Campus, Goa, India \\ Correspondence should be addressed to Abhishek Joshi; abhijoshi2008@gmail.com
}

Received 24 November 2016; Accepted 11 January 2017; Published 16 February 2017

Academic Editor: Arun K. Sangaiah

Copyright ( 2017 Abhishek Joshi et al. This is an open access article distributed under the Creative Commons Attribution License, which permits unrestricted use, distribution, and reproduction in any medium, provided the original work is properly cited.

\begin{abstract}
Wireless Sensor Network consists of large number of nodes densely deployed in ad hoc manner. Usually, most of the application areas of WSNs require two-dimensional (2D) topology. Various emerging application areas such as airborne networks and underwater wireless sensor networks are usually deployed using three-dimensional (3D) network topology. In this paper, a static 3D cluster-based network topology has been proposed for airborne networks. A network protocol stack consisting of various protocols such as TDMA MAC and dynamic routing along with services such as time synchronization, Cluster Head rotation, and power level management has been proposed for this airborne network. The proposed protocol stack has been implemented on the hardware platform consisting of number of TelosB nodes. This 3D airborne network architecture can be used to measure Air Quality Index (AQI) in an area. Various parameters of network such as energy consumption, Cluster Head rotation, time synchronization, and Packet Delivery Ratio (PDR) have been analyzed. Detailed description of the implementation of the protocol stack along with results of implementation has been provided in this paper.
\end{abstract}

\section{Introduction}

Wireless Sensor Network (WSN) is an ad hoc network consisting of large number of nodes distributed in the area of interest in predefined or random manner. WSNs are tightly constrained in terms of computing power, communication range, and energy which also makes unique differentiating factor in the way of operation of the network from rest of the conventional ad hoc networks [1]. A node in WSN consists of computing unit, communication unit, and sensing unit coupled with power supply [2]. Nodes in WSNs are largely dependent on batteries as the primary source of energy.

WSNs can be characterized into different categories depending on their application areas such as military applications [3], environmental applications [4], and home and industrial applications [5]. WSNs play vital part in military applications where they are used for monitoring friendly forces, equipment, ammunition, and surveillance of the battlefield. WSNs are also integral part of environmental applications where they are used for wildlife monitoring, environmental condition monitoring, precision agriculture, forest fire detection, flood detection, air pollution monitoring, and so on. Home and commercial industries use WSNs in home automation, structure monitoring, inventory management, factory process control, theft detection, vehicle tracking, and so on.

Typically, most of the application areas covered by WSNs take into account only 2D topology of the network which includes $x$ and $y$ planes in the network. But there are crucial emerging areas such as airborne networks [6] and underwater networks [7] which take into account the depth or height $(z)$ parameter of the network as well. Characteristics of airborne networks are high mobility, high bit error rate, and intermittent connectivity [8]. Underwater networks encounter large and variable propagation delay, severe Doppler phenomenon, and multipath effects [9]. Since the depth or height parameter has to be included in 3D WSN, conventional protocols of 2D WSN cannot fulfill the requirements of these application areas. Hence, there is a need for developing protocol stack for 3D WSNs which addresses unique features and challenges of these networks. Air quality monitoring is an application of 3D WSN. In this application, a 3D airborne network has to be deployed to monitor the quality of the air. Particulate Matter (PM), ground level ozone, carbon monoxide, nitrogen oxide, 
sulfur oxide, and lead are primary sources of air pollutants. They are also called criteria pollutants. These pollutants are required to be monitored for calculating Air Quality Index (AQI) [10]. AQI for an area tells us how healthy the air is to breathe.

Recently authors have proposed network protocol stack for 3D WSN in [11]. This protocol stack has been implemented using Cooja simulation platform. In our work, protocol stack proposed in [11] has been implemented on static 3D airborne wireless sensor network using hardware platform. This platform consists of number of TelosB nodes deployed at various heights in the form of clusters. These clusters consist of a Cluster Head $(\mathrm{CH})$ node and number of Cluster Nodes $(\mathrm{CN})$. Along with this, a single node deployed on the ground has been used as Base Station. Nodes in the network communicate with Base Station (BS) via their respective $\mathrm{CH}$ nodes in multihop manner. A complete protocol stack consisting of TDMA MAC and dynamic routing along with services such as time synchronization, power level management, and Cluster Head rotation has been deployed on this 3D airborne network. This hardware implementation of complete protocol stack along with its results and analysis has been discussed in this paper.

The rest of this paper is organized as follows. In Section 2, literature review of various protocols of WSNs such as time synchronization and medium access control has been discussed. A lightweight energy efficient time synchronization protocol proposed by authors Tian et al. in [12] has also been discussed in detail in this section. Proposed 3D cluster-based network architecture of airborne network has been discussed in Section 3. In Section 4, operation of the proposed protocol stack on 3D network has been described in detail. In Section 5 results of this implementation along with analysis of this result have been provided. Conclusions have been provided in Section 6.

\section{Literature Survey}

In this section, survey of various protocols such as time synchronization and medium access protocols has been discussed. In Section 2.1, description of time synchronization protocols has been provided and in Section 2.2, description of MAC protocols has been provided.

2.1. Time Synchronization Protocols. Time synchronization is a process of achieving a common clock among network nodes [13]. The clock at each node consists of timer circuitry, which is based on crystal oscillators giving a local time to every node. The time in a node's clock is basically a counter that gets incremented with crystal oscillators. The interrupt handler must increment the clock by one unit each time an interrupt occurs. Clock $C(t)$ can be realized with the help of the following equation:

$$
C(t)=\alpha t+\beta,
$$

where $\alpha$ is a skew and $\beta$ is an offset of the clock.

Clock skew is defined as the rate of deviation of a clock from the true time and clock offset is defined as the difference between the time reported by a clock and the real time. For the perfect clock, $\alpha=1$ and $\beta=0$.

To achieve the common clock, nodes in the network synchronize their local clocks with each other and also with the reference clock. Time synchronization is an important service for various WSN applications such as event detection [14], environment monitoring [15], and target tracking [16]. It is also required for medium access control, coordinated sensing, and multinode cooperative communication. It plays a vital role in saving energy in WSN since it provides the possibility of utilizing sleep-wake cycle in the network [17].

Traditional methods of time synchronization in ordinary networks are not applicable in the sensor network since they are complex in nature and consume more power. Network Time Protocol (NTP) is preferred clock synchronization technique used by time servers operating in the Internet [18]. It provides coordinated universal time (UTC). NTP uses levels of clock servers for the purpose of synchronization but it is not suitable in wireless sensor networks, since it consumes higher amount of energy. In addition, it uses GPS which is an expensive hardware for a low-cost device. GPS may not be available everywhere such as inside buildings or under water. Commonly used time synchronization protocols in WSN include Reference-Broadcast Synchronization (RBS), Timing-sync Protocol for Sensor Networks (TPSN), and Flooding Time Synchronization Protocol (FTSP).

RBS is a broadcast protocol which makes use of receiver to receiver synchronization scheme [19]. The designated root node broadcasts a beacon. Multiple nodes receive this beacon in concurrent manner. Nodes find out their local time at the time of reception of beacon message and then they compare their local time with adjacent nodes. This comparison leads to calculation of phase offset. Main advantage of RBS is that this protocol removes the nondeterminism from the sender. One of the major disadvantages is that it is not suitable for large multihop networks.

TPSN is a sender to receiver synchronization [20]. Working of this protocol is divided into two phases. First phase is called the level discovery phase. Second phase is the synchronization phase. In the level discovery phase, a level is assigned to each node. Root node is considered to be at level zero. In the synchronization phase, nodes from level one initiate the two-way messaging with the root node. This process iterates throughout the network. Root node is the originator of both phases. TPSN provides improvement of precision over RBS protocol. The major disadvantage is that it does not allow dynamic topology changes.

FTSP is another sender to receiver synchronization protocol [21]. FTSP broadcasts timing information to all nodes that are able to receive the message. Those nodes which receive the information message from the root node calculate their offset from the global time. These nodes also calculate their clock skew using linear regression. FTSP is robust in compensating node and link failures. It also allows for a dynamic topology but FTSP is not suitable for large multihop networks.

All of the above-discussed protocols exchange large number of messages during the synchronization phase. For achieving long lifetime of deployed network, minimizing the energy footprint is the primary goal in designing WSN. Time 
TABLE 1: Message exchange comparison for time synchronization protocols.

\begin{tabular}{lcc}
\hline Protocol & \multicolumn{2}{c}{ Number of message exchanges } \\
& Nodes $=50$ & Nodes $=250$ \\
\hline TPSN & 1000 & 5000 \\
RBS & 1250 & 30000 \\
FTSP & 500 & 2500 \\
\hline
\end{tabular}

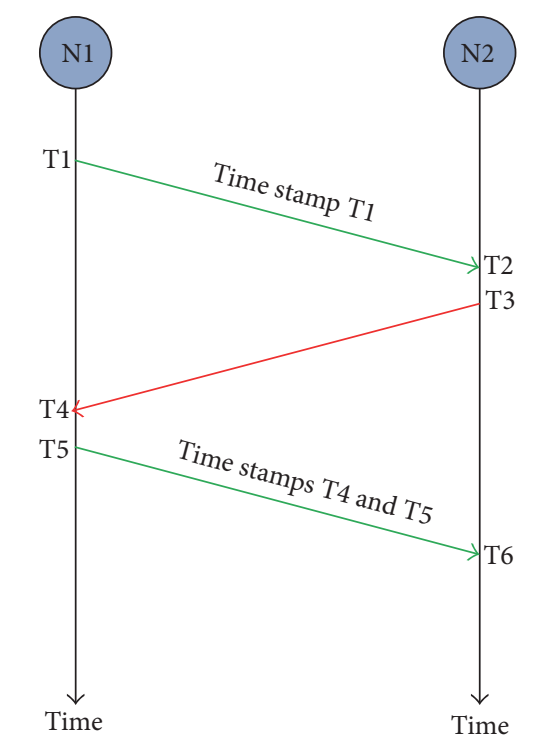

Figure 1: Tri-Message time synchronization.

synchronization protocols for WSN cannot be excluded from this constraint. Authors Noh et al. have conducted simulation of above-discussed time synchronization protocols on the basis of number of message exchanges for various numbers of nodes [22]. These results for TPSN, RBS, and FTSP have been tabulated in Table 1.

Recently, a time synchronization protocol has been proposed for underwater acoustic sensor networks. This protocol termed as Tri-Message synchronization protocol is suitable for high latency and resource constrained underwater networks. A brief description of Tri-Message protocol has been provided in following subsection.

2.1.1. Tri-Message Time Synchronization Protocol. In TriMessage synchronization there are two nodes, Node N1 and Node N2. N1 is considered to be the originator of Tri-Message synchronization and assumed to have synchronization with the global clock. Figure 1 represents Tri-Message time synchronization process.

In the Tri-Message synchronization phase, N1 transmits first message to N2 at time T1. This message includes the time stamp T1. N2 receives this message at time T2. Later, N2 sends the message back to $\mathrm{N} 1$ at time T3, which is received by $\mathrm{N} 1$ at time T4. Finally, N1 transmits the third message at time T5. In this message, $\mathrm{N} 1$ includes the time stamps T4 and T5. N2 receives this message from $\mathrm{N} 1$ at time $\mathrm{T} 6$, which completes the Tri-Message synchronization process. After the completion of this process, N2 has all six time stamps available with it. Time gap between T2 and T3 is considered to be processing time for N2. Similarly, equal duration gap between T4 and T5 is the processing time for N1. Clock skew $(\alpha)$ and offset $(\beta)$ in the Tri-Message synchronization protocol can be calculated with following equations:

$$
\begin{aligned}
& \alpha=\frac{T 6-T 2}{T 5-T 1}, \\
& \beta=\frac{T 2+T 3}{2}-\alpha \frac{T 4+T 5}{2} .
\end{aligned}
$$

In this way, the Tri-Message time synchronization protocol requires only three message exchanges to achieve time synchronization between two nodes. Hence for time synchronization of 50 nodes, it will require 150 message exchanges whereas for 250 nodes, 750 messages will be exchanged. Thus compared to TPSN, RBS, FTSP, and other linear regression based time synchronization protocols, Tri-Message protocol is a lightweight time synchronization protocol. Depending on advantage of energy efficiency, a Tri-Message time synchronization protocol has been utilized in this work. An extension of this protocol has been proposed for multihop topology and has been implemented on 3D airborne network. For achieving higher accuracy in time synchronization, Tri-Message protocol can be replaced with fine grained time synchronization protocols.

In the next subsection, we provide review of medium access protocols.

2.2. Medium Access Control Protocols. MAC (Medium Access Control) layer plays important role in WSN protocol to provide the reliability and efficiency. MAC protocols are responsible for channel access policies, scheduling, buffer management, and error control [23]. The medium access control protocols for the wireless sensor networks can be classified broadly into two categories [24]:

(1) Contention based MAC protocols.

(2) Schedule based MAC protocols.

In contention based MAC protocols successful transmission cannot be assured. In these protocols as per requirement, all nodes in the network make use of a single common channel. This method differs from the schedule based MAC protocols in which the channel is further divided and preallocated for all the nodes in the network. In contention based MAC protocols, whenever the node wants to transmit its data, contention mechanism decides whether the node has the privilege to access the common channel [25]. These protocols are advantageous over schedule based MAC protocols in terms of network scalability and traffic in the network. Also they are not largely affected by topology changes in the network. The major drawback of contention based MAC protocols is that they utilize the energy of the network in ineffective manner [26].

Schedule based MAC protocols help in avoiding overhearing, idle listening, and collisions by scheduling transmit and listen periods. Time synchronization is basic requirement for schedule based MAC protocols [27]. These protocols 
demand stringent time synchronization requirements. The categories of schedule based protocols are as follows:

(1) Time Division Multiple Access (TDMA).

(2) Frequency Division Multiple Access (FDMA).

(3) Code Division Multiple Access (CDMA).

In TDMA protocol, a common channel is utilized by all the nodes in the network which is divided into time slots [28]. The advantage of TDMA protocol is that network has no data collision. Since the time slots are predefined, nodes turn on and off their radios at predefined time which further saves energy in the network. A major drawback of TDMA protocol is the stringent requirement of time synchronization in the network [26]. In FDMA protocol all the nodes in the network use same time slot but they operate at different carrier frequencies. This scheme also avoids data collision in the network but at the cost of additional hardware [29]. CDMA protocol makes use of spread-spectrum technique along with the coding scheme. This allows multiple users to be multiplexed over the same physical channel. This scheme also avoids data collision in the network but requires additional computing requirement resulting into increased energy consumption which is contradictory with the basic requirement for WSNs [30].

Among various schedule based protocols such as FDMA, CDMA, and TDMA based protocols, TDMA based MAC protocols can exploit advantages in terms of simplicity, fairness, and energy efficiency. Collisions, idle listening, and overhearing can be avoided in this protocol. Hidden node problem is easily solved without using an extra message overhead because neighboring nodes transmit at different time slots. Channel utilization can be significantly improved if parallelism in communication is allowed. These concurrent transmission schemes can be exploited using proper channel reuse concept already available in cellular mobile networks. Slots can be reserved for future expansion and can be allocated on dynamic basis to address the scalability issue. If we assume structured deployment and deterministic scheduling scheme with known traffic patterns, then any simple or energy efficient time synchronization can suffice in TDMA MAC protocol.

Authors Handy et al. proposed Low Energy Adaptive Clustering Hierarchy (LEACH) protocol [31]. This protocol is based on TDMA based MAC protocol. Operation of protocol is divided in two phases. In first phase, clusters are organized. During second phase, data from all the nodes in the network gets transferred to the Base Station node. Originally LEACH protocol was developed for 2D WSNs. Authors Baghouri et al. translated existing LEACH protocol for 3D WSNs while retaining its original features [32].

In this work, a new variant of TDMA based MAC proto$\mathrm{col}$ has been proposed for $3 \mathrm{D}$ airborne networks. This protocol has been implemented as a part of protocol stack on hardware platform of 3D airborne network. In Section 3, architecture of 3D airborne network has to be described in detail.

\section{Network Architecture of 3D Airborne Wireless Sensor Network}

In this work, a static and structured 3D network architecture has been proposed. This network has been divided into $L$ levels with each level separated by the vertical distance of $y \mathrm{~m}$. At every level, a cluster of $n$ nodes has been deployed. This cluster consists of a Cluster Head node and number of Cluster Member Nodes. The nodes of cluster have been deployed within the diameter of $x \mathrm{~m}$. These clusters have been deployed vertically above each other. At ground level, a single node has been deployed which serves as the Base Station in the network. In Figure 2, the proposed network architecture has been illustrated.

Nodes in the network are preliminary divided into three categories:

(1) Base Station (BS) node.

(2) Cluster Head $(\mathrm{CH})$ node.

(3) Cluster Member (CN) node.

Base Station (BS) node deployed at ground level is used to send control information to the nodes in the network. Similarly, data sensed from the nodes can be collected at the BS. Initially, the BS node sends the control information to the $\mathrm{CH}$ node situated one level above it. After receiving the control information from BS node, this $\mathrm{CH}$ node transmits information to the $\mathrm{CH}$ node in the level above it. Once the control information has been received by all the $\mathrm{CH}$ nodes, these $\mathrm{CH}$ nodes send this control information to their respective $\mathrm{CN}$ nodes. $\mathrm{CN}$ nodes can then transmit the sensed data information to their respective $\mathrm{CH}$ nodes. Every $\mathrm{CH}$ node aggregates the collected data information and forwards it in hop by hop manner to the BS.

Node addressing scheme has also been illustrated in Figure 2. A node is represented as the address NLxx where $L$ stands for the level number and nn stands for the node number. For example, BS node at level 0 is represented as N000. At level 1, nodes are represented as N100 to N1xx wherein N100 is initially deployed as CH node. Similarly at level 2 nodes are addressed from N200 to N2xx, wherein $\mathrm{N} 200$ is initially deployed $\mathrm{CH}$ node.

This 3D network architecture has been tested using hardware platform. Hardware implementation of the network has been carried out on TelosB nodes. TelosB node is based on $8 \mathrm{MHz}$ TI MSP430 microcontroller with $10 \mathrm{kB}$ of RAM. It has $250 \mathrm{kbps} 2.4 \mathrm{GHz}$ IEEE 802.15 .4 wireless transceiver. ADC, DAC, supply voltage supervisor, and DMA controller are integrated into the node. Also, the node has integrated humidity, temperature, and light sensors. TelosB supports ultra-low mode current consumption and it has support for variable transmission power which suits the network requirement in which the packet has to be transmitted at different distances. In this work, for implementation of network architecture on hardware platform, total 3 levels have been deployed. At ground level, BS node has been deployed. At other two levels, 5 nodes each have been deployed. Vertical distance between clusters has been taken as $6 \mathrm{~m}$ whereas the diameter of cluster at each level has been taken as $4 \mathrm{~m}$. 


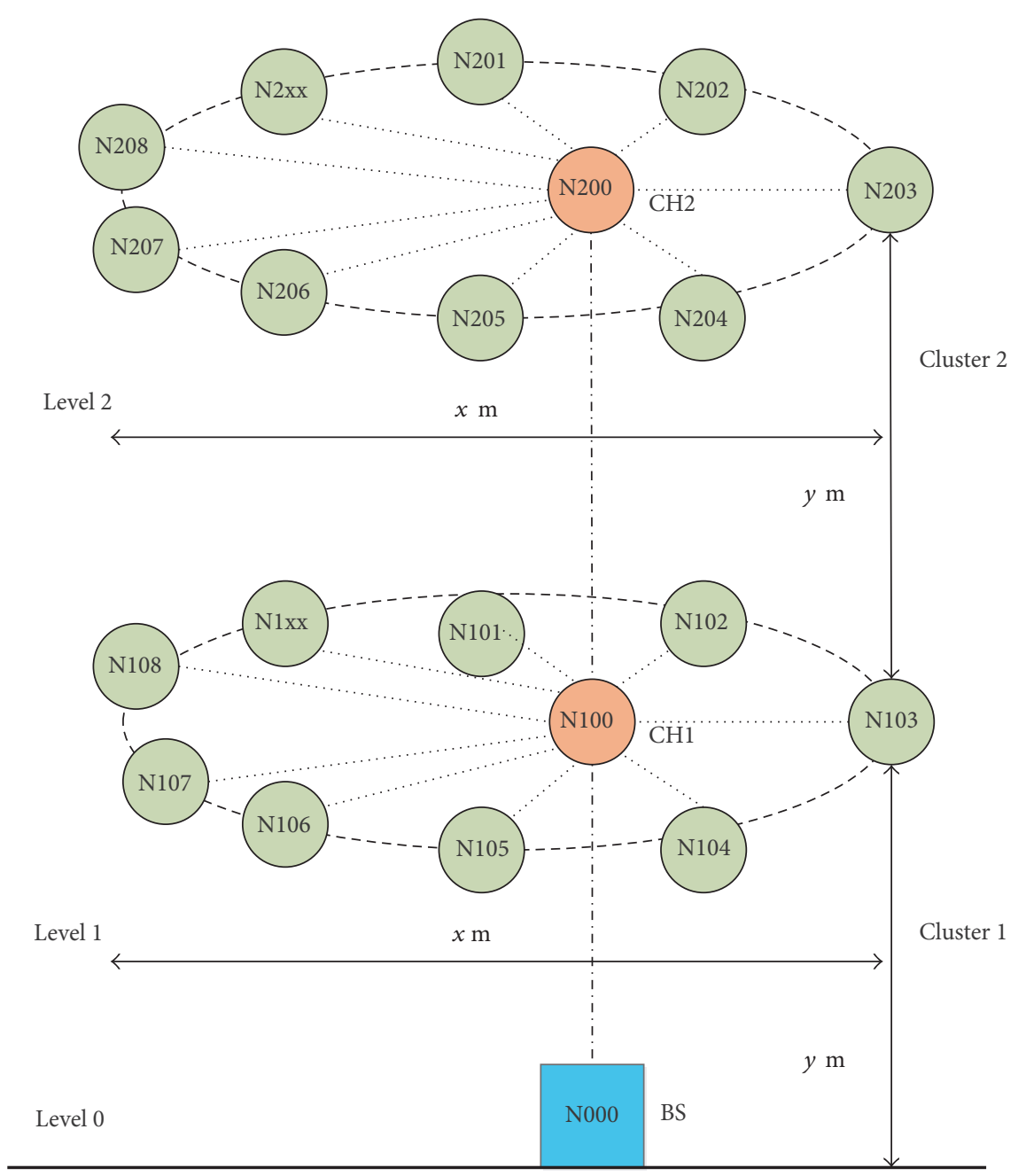

FIGURE 2: Network architecture of 3D airborne network.

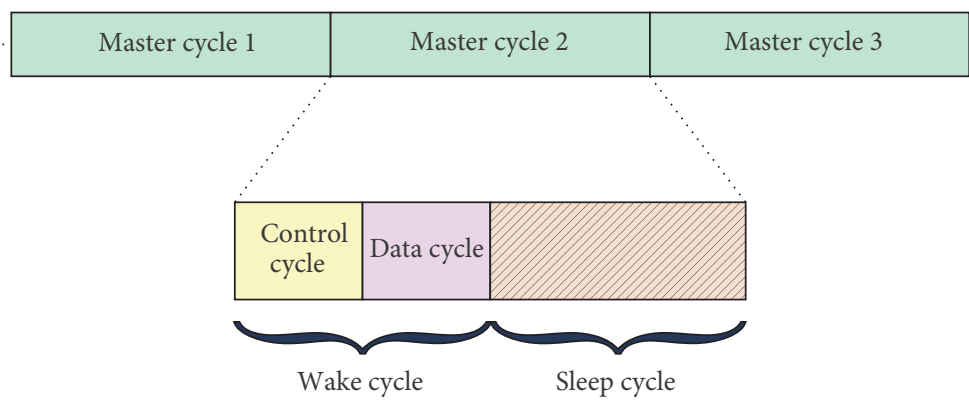

Figure 3: Composition of master cycle.

\section{Working of the Proposed Protocol Stack}

In this work, a variant of TDMA based MAC protocol termed as Dynamic Cluster-Based TDMA MAC (DCBTDMA MAC) protocol has been proposed and implemented on 3D airborne network. In this protocol, network operation is divided into periodic cycles. These periodic cycles have been termed as master cycles. Every master cycle is divided into sleep and wake cycle. Wake cycle is further subdivided into control and data cycle.

Figure 3 represents composition of master cycle.

Wake cycle begins with control cycle. Time synchronization takes place during control cycle. In control cycle, a multihop version of Tri-Message time synchronization has been used. Description of proposed multihop version has been given in the following subsection. 


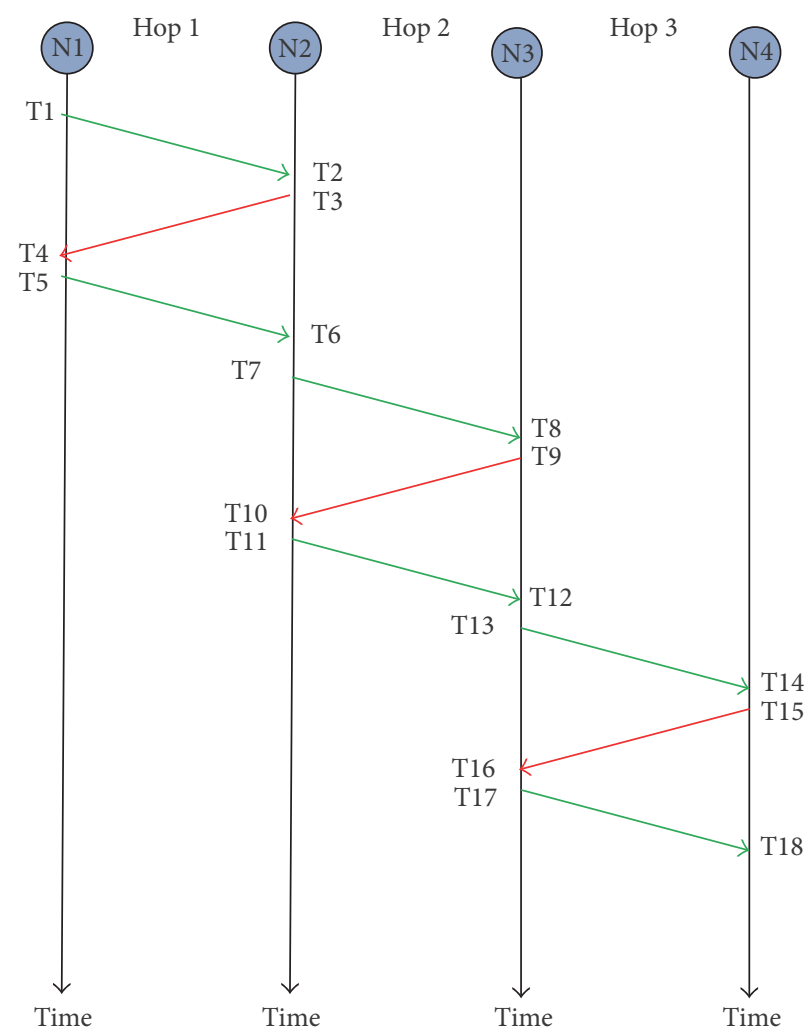

FIgURE 4: Multihop Tri-Message time synchronization.

4.1. Multihop Tri-Message Time Synchronization. In this work, a multihop version of Tri-Message time synchronization protocol has been proposed which is illustrated in Figure 4. Node N1 acts as a reference clock source in the network. Node N2 in hop 1 synchronizes its clock with reference to Node N1 after three messages exchange. At the end of time instance T6, clock of Node N2 gets synchronized with respect to Node N1. At time instant T7, Node N2 initiates time synchronization process with Node N3 on hop 2. Three messages are exchanged between Node N2 and Node N3. At the end of time instance T12, Node N3 gets synchronized with Node N2. Same process is repeated until all the nodes in the network are time synchronized with respect to the reference clock in the network. Considering three-hop structure of the network, the corrected clock at Node N3 can be calculated as follows:

For hop 1

$$
C_{1}(t)=\alpha_{1} t+\beta_{1}
$$

For hop 2

$$
C_{2}(t)=\alpha_{2} t+\beta_{2}
$$

For hop 3

$$
C_{3}(t)=\alpha_{3} t+\beta_{3} \text {. }
$$

Using (3), (4), and (5) clock of Node N3 can be calculated as

$$
t=\frac{C_{3}(t)-\beta_{3}-\alpha_{2} \alpha_{3} \beta_{1}-\alpha_{3} \beta_{2}}{\alpha_{1} \alpha_{2} \alpha_{3}} .
$$

4.2. Dynamic Cluster-Based TDMA MAC. During hardware implementation of the protocol, a fourth message has been added in the Tri-Message time synchronization protocol. In this message, clock skew and offset values have been broadcasted by the synchronized node. This message has been used to validate the values of skew $(\alpha)$ and offset $(\beta)$ for the node. Thus, Tri-Message time synchronization requires exchange of 4 messages between two nodes. Considering one time slot for every message, in every control cycle, 4 time slots have been utilized for time synchronization of two nodes.

In our hardware implementation of 3D airborne network, multihop Tri-Message time synchronization has been used in the following manner. Base Station node (N000) initiates time synchronization in the network. Initially, N000 synchronizes $\mathrm{CH}$ node in cluster 1 (N100). After getting synchronized with N000, N100 synchronizes CH node of cluster 2 (N200). This completes synchronization of all the $\mathrm{CH}$ nodes in the network in hop by hop manner through vertical communication link. When all the $\mathrm{CH}$ nodes in the network are time synchronized with BS, CH nodes begin the process of time synchronization in their respective clusters. The time synchronization between $\mathrm{CH}$ nodes and their respective $\mathrm{CN}$ nodes takes place over horizontal communication link. In the network, dynamic power levels have been used for the transmission. For example, in hardware implementation, two different levels have been utilized for horizontal and vertical communication links. While communicating over a vertical link of $6 \mathrm{~m}$ distance, $\mathrm{CH}$ nodes use higher power level of transmission. On the other hand, to communicate over the horizontal 


\begin{tabular}{|c|c|c|c|c|c|c|}
\hline $\begin{array}{c}\text { TS1 } \\
\text { to } \\
\text { TS4 }\end{array}$ & $\begin{array}{l}\text { TS5 } \\
\text { to } \\
\text { TS8 }\end{array}$ & $\begin{array}{l}\text { TS9 } \\
\text { to } \\
\text { TS12 }\end{array}$ & $\begin{array}{l}\text { TS13 } \\
\text { to } \\
\text { TS16 }\end{array}$ & $\begin{array}{l}\text { TS17 } \\
\text { to } \\
\text { TS20 }\end{array}$ & $\begin{array}{l}\text { TS21 } \\
\text { to } \\
\text { TS24 }\end{array}$ & TS25-TS44 \\
\hline $\begin{array}{c}\text { BS } \\
\text { (N000) } \\
\text { to } \\
\text { CH1 } \\
\text { (N100) }\end{array}$ & $\begin{array}{c}\mathrm{CH} 1 \\
(\mathrm{~N} 100) \\
\text { to } \\
\mathrm{CH} 2 \\
(\mathrm{~N} 200)\end{array}$ & $\begin{array}{c}\text { CH1 } \\
\text { (N100) } \\
\text { to } \\
\text { CN1 } \\
\text { (N101) }\end{array}$ & $\begin{array}{c}\mathrm{CH} 1 \\
\text { (N100) } \\
\text { to } \\
\mathrm{CN} 2 \\
\text { (N102) }\end{array}$ & $\begin{array}{c}\mathrm{CH} 1 \\
\text { (N100) } \\
\text { to } \\
\mathrm{CN} 3 \\
\text { (N103) }\end{array}$ & $\begin{array}{c}\mathrm{CH} 1 \\
\text { (N100) } \\
\text { to } \\
\mathrm{CN} 4 \\
\text { (N104) }\end{array}$ & $\begin{array}{l}\text { Future expansion of } \\
\qquad 5 \text { nodes }\end{array}$ \\
\hline & & $\begin{array}{c}\mathrm{CH} 2 \\
\text { (N200) } \\
\text { to } \\
\mathrm{CN} 1 \\
\text { (N201) }\end{array}$ & $\begin{array}{c}\mathrm{CH} 2 \\
(\mathrm{~N} 200) \\
\text { to } \\
\mathrm{CN} 2 \\
(\mathrm{~N} 202)\end{array}$ & $\begin{array}{c}\mathrm{CH} 2 \\
\text { (N200) } \\
\text { to } \\
\mathrm{CN} 3 \\
\text { (N203) }\end{array}$ & $\begin{array}{c}\mathrm{CH} 2 \\
(\mathrm{~N} 200) \\
\text { to } \\
\mathrm{CN} 4 \\
\text { (N204) }\end{array}$ & $\begin{array}{c}\text { Future expansion of } \\
5 \text { nodes }\end{array}$ \\
\hline
\end{tabular}

Figure 5: Control cycle structure.

\begin{tabular}{|c|c|c|c|c|c|c|}
\hline TS45 & TS46 & TS47 & TS48 & TS49-TS53 & TS54 & TS55 \\
\hline $\begin{array}{c}\mathrm{CN} 1 \\
\text { (N101) } \\
\text { to } \\
\mathrm{CH} 1 \\
(\mathrm{~N} 100)\end{array}$ & $\begin{array}{c}\mathrm{CN} 2 \\
\text { (N102) } \\
\text { to } \\
\mathrm{CH} 1 \\
\text { (N100) }\end{array}$ & $\begin{array}{c}\mathrm{CN} 3 \\
\text { (N103) } \\
\text { to } \\
\mathrm{CH} 1 \\
\text { (N100) }\end{array}$ & $\begin{array}{c}\mathrm{CN} 4 \\
(\mathrm{~N} 104) \\
\text { to } \\
\mathrm{CH} 1 \\
(\mathrm{~N} 100)\end{array}$ & $\begin{array}{l}\text { Future expansion of } \\
5 \text { nodes }\end{array}$ & $\begin{array}{c}\mathrm{CH} 2 \\
\text { (N200) } \\
\text { to } \\
\mathrm{CH} 1 \\
(\mathrm{~N} 100)\end{array}$ & $\begin{array}{c}\mathrm{CH} 1 \\
\text { (N100) } \\
\text { to } \\
\text { BS } \\
(\mathrm{N} 000)\end{array}$ \\
\hline $\begin{array}{c}\mathrm{CN} 1 \\
\text { (N201) } \\
\text { to } \\
\mathrm{CH} 2 \\
(\mathrm{~N} 200)\end{array}$ & $\begin{array}{c}\mathrm{CN} 2 \\
\text { (N202) } \\
\text { to } \\
\mathrm{CH} 2 \\
\text { (N200) }\end{array}$ & $\begin{array}{c}\mathrm{CN} 3 \\
\text { (N203) } \\
\text { to } \\
\mathrm{CH} 2 \\
\text { (N200) }\end{array}$ & $\begin{array}{c}\mathrm{CN} 4 \\
\text { (N204) } \\
\text { to } \\
\mathrm{CH} 2 \\
\text { (N200) }\end{array}$ & $\begin{array}{l}\text { Future expansion of } \\
5 \text { nodes }\end{array}$ & & \\
\hline
\end{tabular}

FIGURE 6: Data cycle structure.

distance of $2 \mathrm{~m}$, nodes use lower power level of transmission. Use of different power levels in the network helps in achieving parallel communication among the horizontal communication links in the network. $\mathrm{CH}$ at different levels communicate with their $\mathrm{CN}$ nodes from their respective clusters in same time slots. Parallel communication of $\mathrm{CH}$ nodes with their respective $\mathrm{CN}$ nodes helps in optimizing the time slot (TS) requirement of the network. Figure 5 represents the structure of the control cycle phase of network operation.

Multihop Tri-Message time synchronization protocol has been used in our network which requires 4 time slots for synchronizing two nodes in the network. In first four time slots TS1-TS4, N100 synchronizes with N000. In TS5-TS8, N200 gets synchronized with N100. By the end of TS8, CH nodes in the network are time synchronized with BS. From TS9 onwards, $\mathrm{CH}$ nodes start synchronizing CN nodes of their respective clusters. There are 5 nodes in every level with one among these nodes acting as a $\mathrm{CH}$ at any given point of time. There are $4 \mathrm{CN}$ nodes in every cluster. Time synchronization of different $\mathrm{CN}$ nodes from different levels with their respective $\mathrm{CH}$ nodes takes place in concurrent manner. Total 16 time slots are utilized for synchronizing N101-N104 with N100 in level 1 and N201-N204 with N200 in level 2. Time synchronization of all the nodes is achieved by the end of TS24. In proposed network protocol stack, we have made the provision of dynamically adding 5 nodes in each level to scale the network. Extra 20 time slots are reserved for dynamic addition of nodes in the network. At TS45, control cycle is complete and from subsequent time slot onwards, the data cycle starts. If no new nodes are added, duration of TS25-TS44 is used as sleep period.

During data cycle, $\mathrm{CN}$ nodes transmit their sensed data to their respective $\mathrm{CH}$ nodes in preallotted time slot over horizontal communication link. For communication over horizontal link, lower power level of transmission is utilized by $\mathrm{CN}$ nodes. These lower power levels permit the parallel intracluster communication. When $\mathrm{CN}$ nodes of cluster 2 transmit their data to the $\mathrm{CH}$ node of cluster 2, the $\mathrm{CN}$ nodes of cluster 1 parallel transmit the data to the $\mathrm{CH}$ node of cluster 1 . When $\mathrm{CH}$ nodes receive the data, it is aggregated at respective $\mathrm{CH}$ nodes. After this aggregation, the forwarding of data is initiated over vertical communication link. $\mathrm{CH}$ node of cluster 2 forwards the aggregated data to the $\mathrm{CH}$ node of cluster 1. Further $\mathrm{CH}$ node of cluster 1 appends his own data and forwards it to the BS.

Figure 6 represents the structure of the data cycle phase. In Figure 6, N101 transmits its sensed data to N100 in TS46. Because of utilization of dynamic power levels, N201 also transmits its sensed data to N200 in the same TS. Similarly, N102-N104 transmit their sensed data to N100 in TS46TS48. N202-N204 also transmit their sensed data to N200 in the same time slts. Extra 5 slots, TS49-TS53, have been reserved for adding extra 5 nodes in each level. In TS54, N200 transmits its aggregated data to N100 over a vertical communication link and finally in TS55, N100 transmits its aggregated data along with data received from N200 to N000. By the end of TS55, the network operation for one wake cycle is complete. After wake cycle nodes in the network sleep which 
has the same duration of wake cycle, the combined sleep and wake cycle constitute a master cycle. These master cycles repeat in periodic manner throughout the network lifetime.

As discussed earlier in this section, provision of dynamic addition of nodes has been made during control cycle as well as data cycle. Up to 5 nodes can be added in each level at any point of time. The dynamic node addition is controlled by BS. Certain parameters have to be communicated with BS such as number of nodes which have to be added, the levels in which they have to be added, and the master cycle number during which they have to be added. After this information has been externally fed to the BS, it includes this information in control cycle to communicate with respective $\mathrm{CH}$ nodes. After receiving the control information about the node addition, $\mathrm{CH}$ nodes communicate with the newly added nodes in the predefined master cycle. During control cycle, $\mathrm{CH}$ nodes share their timing information for synchronization of the clocks of the newly added nodes and during data cycles, $\mathrm{CH}$ nodes collect the sensed data from these newly added nodes which make these nodes as a part of the network.

We have also introduced Cluster Head rotation policy in our network to balance the energy in the network. $\mathrm{CH}$ nodes at each level communicate with other $\mathrm{CH}$ nodes above and below their level over vertical communication link utilizing higher power level of transmission. Also $\mathrm{CH}$ nodes communicate with $\mathrm{CN}$ nodes in their respective clusters over horizontal communication link utilizing lower power level of transmission. Because of all these communications, $\mathrm{CH}$ nodes consume higher amount of energy compared to $\mathrm{CN}$ nodes. If the node acts as a $\mathrm{CH}$ node for prolonged duration of time, higher amount of energy would be depleted from this $\mathrm{CH}$ node leading to complete drainage of battery. This might result into dead node which would hamper the operation of the network. To avoid the network failure and to balance energy in the network, a $\mathrm{CH}$ rotation policy has been implemented which takes into account the depleted energy as the criteria for electing new $\mathrm{CH}$. During hardware implementation of the network, at every fifth master cycle, $\mathrm{CN}$ nodes transmit their depleted energy levels along with sensed data to their respective $\mathrm{CH}$ nodes. $\mathrm{CH}$ nodes locally elect the next $\mathrm{CH}$ for its level and transmit the information to the subsequent $\mathrm{CH}$ node until this information is available with BS. During sixth master cycle, $\mathrm{CH}$ nodes percolate the information about newly elected $\mathrm{CH}$ within their respective clusters during control cycle phase. From seventh master cycle onward, the newly elected $\mathrm{CH}$ takes effect. This process of Cluster Head election iterates periodically throughout the span of the network.

In LEACH clustering protocol for 2D as well as 3D WSNs, $\mathrm{CH}$ nodes communicate with BS node in single hop manner. This results into increased energy consumption of $\mathrm{CH}$ nodes since every $\mathrm{CH}$ node has to communicate with $\mathrm{BS}$ node in direct manner. This technique requires increased amount of energy for communication compared to our 3D airborne protocol stack where $\mathrm{CH}$ nodes communicate with BS node in multihop manner. This reduced energy consumption is helpful in prolonging lifetime of the network.
TABLE 2: Design parameters of deployed 3D airborne network.

\begin{tabular}{lc}
\hline Network parameter & Value \\
\hline$L$ & 3 \\
$y$ & $6 \mathrm{~m}$ \\
$x$ & $4 \mathrm{~m}$ \\
$n$ & 5 \\
\hline
\end{tabular}

Authors Attarzadeh and Mehrani proposed a new threedimensional clustering method for WSN [33]. In their proto$\mathrm{col}$, the network is considered static and $\mathrm{CH}$ nodes are fixed. Cluster Head rotation policy has not been discussed in their protocol. It leads to reduced lifespan of the network compared to our 3D airborne protocol stack since energy for $\mathrm{CH}$ nodes is usually depleted at higher rate compared to rest of the nodes in the network. If no Cluster Head rotation policy is included in the protocol, chances of complete depletion of energy for $\mathrm{CH}$ node increase which results into nonfunctioning of node resulting into reduced lifespan of the network.

Network parameters of the 3D airborne network have been described in the following subsection.

4.3. Network Parameters of Deployed 3D Airborne Network. Design parameters of deployed 3D airborne network have been tabulated in Table 2 .

Packet Delivery Time is composed of Transmission Time (TT) and Propagation Delay (PD) in the network.

Transmission Time (TT) is defined as ratio of Packet Size (PS) to Bit Rate (BR)

$$
\mathrm{TT}=\frac{\mathrm{PS}}{\mathrm{BR}} .
$$

Propagation Delay (PD) is ratio of Distance to Speed

$$
\mathrm{PD}=\frac{\text { Distance }}{\text { Speed }} \text {. }
$$

Hence

$$
\begin{aligned}
& \text { Packet Delivery Time (PDT) } \\
& =\text { Transmission Time (TT) } \\
& + \text { Propagation Delay (PD) }
\end{aligned}
$$

Time slot for transmission and reception of the node can be calculated from PDT. In our implementation, we have taken time slot values larger than the PDT value considering an application involving data collection at regular intervals. Delay in this network can be reduced significantly by taking time slot values of duration equal to PDT, which can be the optimum values for time slot duration.

As discussed in Section 4, we have made use of dynamic power levels for transmissions. On vertical communication link, higher power level of transmission has been used. A transmission circuitry consumes $I_{\mathrm{TX}}$ during higher power level of transmission. On horizontal communication link, lower power level of transmission has been used. Transmission circuitry for horizontal link consumes $I_{\mathrm{TX}_{\mathrm{PL}}}$ during lower power level of transmission. 
TABLE 3: Various types of packets used in the network.

\begin{tabular}{lcc}
\hline Packet type & Link type & Size \\
\hline Time sync & Vertical & 27 bytes \\
Time sync & Horizontal & 29 bytes \\
Data & Vertical & 23 bytes \\
Data & Horizontal & 23 bytes \\
Data + energy & Vertical & 24 bytes \\
Data + energy & Horizontal & 27 bytes \\
\hline
\end{tabular}

Therefore, energy consumed during transmission of packet at higher power level is given as follows:

$$
E_{\mathrm{TX}_{\mathrm{PH}}}=\mathrm{TT} \times V \times I_{\mathrm{TX}_{\mathrm{PH}}} .
$$

Energy consumed during transmission of packet at lower power level is given as follows:

$$
E_{\mathrm{TX}_{\mathrm{PL}}}=\mathrm{TT} \times V \times I_{\mathrm{TX}_{\mathrm{PL}}} .
$$

Energy consumption during reception of packet is uniform across all the nodes in the network. Current requirement for reception is given as $I_{\mathrm{RX}}$.

Hence, energy consumption during reception of packet is as follows:

$$
E_{\mathrm{RX}}=\mathrm{TT} \times V \times I_{\mathrm{RX}} .
$$

\section{Results and Discussion}

During the hardware deployment of 3D airborne network, we have considered various data packets of different sizes. Table 3 gives detailed information about types and sizes for both horizontal and vertical links.

Bit rate of the nodes is set as $20 \mathrm{kbps}$. Time slot duration value is kept $20 \mathrm{~ms}$. Also sleep cycle of $60 \mathrm{~s}$ has been defined during the operation of the network. A new node has been added in level 1 in master cycle 8 and another new node has been included in level 2 in master cycle 9 to represent dynamic node addition feature of our 3D airborne network. Network has been analyzed for multiple master cycles. Multihop Tri-Message time synchronization results have been discussed in Section 5.1. In Section 5.2, energy consumption analysis of network has been provided. Cluster Head rotation policy has been discussed in Section 5.3. In Section 5.4, Packet Delivery Ratio (PDR) for the hardware implementation of the network has been provided.

\subsection{Results of Multihop Tri-Message Time Synchronization} Protocol. Multihop Tri-Message time synchronization takes place during control cycle. Skew and offset are important parameters in our time synchronization protocol. With the help of equation (6), we can correct the local clock of a node in the network with respect to global clock in the network. Considering the network parameters discussed in Section 4.3, results obtained by implementing multihop Tri-Message time synchronization protocol in hardware platform have been presented in Tables 4, 5, and 6.
TABLE 4: Skew and offset values during cycles 1 and 2.

\begin{tabular}{llcc}
\hline Node pair & & Cycle 1 & Cycle 2 \\
\hline \multirow{2}{*}{$000-100$} & Skew & 0.9999 & 0.9999 \\
& Offset & $12008 \mathrm{~ms}$ & $83 \mathrm{~ms}$ \\
\multirow{2}{*}{$100-101$} & Skew & 0.9999 & 0.9999 \\
& Offset & $2089 \mathrm{~ms}$ & $78 \mathrm{~ms}$ \\
\multirow{2}{*}{$100-102$} & Skew & 0.9998 & 0.9999 \\
& Offset & $2061 \mathrm{~ms}$ & $87 \mathrm{~ms}$ \\
\multirow{2}{*}{$100-103$} & Skew & 0.9998 & 1.0010 \\
& Offset & $2171 \mathrm{~ms}$ & $92 \mathrm{~ms}$ \\
$100-200$ & Skew & 1.0010 & 1.0010 \\
& Offset & $1613 \mathrm{~ms}$ & $97 \mathrm{~ms}$ \\
$200-201$ & Skew & 1.0000 & 1.0000 \\
& Offset & $1678 \mathrm{~ms}$ & $94 \mathrm{~ms}$ \\
$200-202$ & Skew & 1.0000 & 0.9999 \\
& Offset & $1762 \mathrm{~ms}$ & $79 \mathrm{~ms}$ \\
$200-203$ & Skew & 1.0030 & 1.0030 \\
& Offset & $1794 \mathrm{~ms}$ & $86 \mathrm{~ms}$ \\
\hline
\end{tabular}

TABLE 5: Skew and offset values during cycles 8 and 9.

\begin{tabular}{lccc}
\hline Node pair & & Cycle 8 & Cycle 9 \\
\hline \multirow{2}{*}{$000-101$} & Skew & 0.9999 & 0.9999 \\
$101-100$ & Offset & $143 \mathrm{~ms}$ & $145 \mathrm{~ms}$ \\
& Skew & 0.9999 & 0.9999 \\
$101-102$ & Offset & $103 \mathrm{~ms}$ & $118 \mathrm{~ms}$ \\
& Skew & 0.9999 & 0.9999 \\
$101-103$ & Offset & $129 \mathrm{~ms}$ & $124 \mathrm{~ms}$ \\
& Skew & 1.0010 & 1.0010 \\
$101-104$ & Offset & $96 \mathrm{~ms}$ & $101 \mathrm{~ms}$ \\
& Skew & 0.9999 & 0.9999 \\
$101-201$ & Offset & $2076 \mathrm{~ms}$ & $69 \mathrm{~ms}$ \\
& Skew & 1.0010 & 1.0010 \\
$201-200$ & Offset & $113 \mathrm{~ms}$ & $110 \mathrm{~ms}$ \\
& Skew & 1.0000 & 1.0000 \\
$201-202$ & Offset & $143 \mathrm{~ms}$ & $144 \mathrm{~ms}$ \\
& Skew & 0.9999 & 0.9999 \\
$201-203$ & Offset & $123 \mathrm{~ms}$ & $128 \mathrm{~ms}$ \\
& Skew & 1.0030 & 1.0030 \\
& Offset & $129 \mathrm{~ms}$ & $138 \mathrm{~ms}$ \\
& Skew & - & 1.0020 \\
& Offset & - & $3174 \mathrm{~ms}$ \\
\hline
\end{tabular}

In Table 4, skew and offset values obtained during hardware implementation of multihop Tri-Message time synchronization protocol in cycle 1 and cycle 2 have been tabulated. N000 is considered to have reference clock for the network. During cycle 1, all the nodes in the network calculate their respective values of skew and offset with help of (2) by comparing their local clocks against the reference clock in the network. Clock correction scheme has been included during the implementation of our network on hardware platform. From cycle 2 onwards the clock correction takes over to correct the 
TABLE 6: Skew and offset (ms) values during cycle 13.

\begin{tabular}{lll}
\hline Node pair & \multicolumn{2}{c}{ Cycle 13 } \\
\hline \multirow{2}{*}{$0-104$} & Skew & 0.9999 \\
\multirow{2}{*}{$104-100$} & Offset & $148 \mathrm{~ms}$ \\
& Skew & 0.9999 \\
$104-101$ & Offset & $136 \mathrm{~ms}$ \\
& Skew & 0.9999 \\
$104-102$ & Offset & $153 \mathrm{~ms}$ \\
\multirow{2}{*}{$104-103$} & Skew & 1.0010 \\
& Offset & $123 \mathrm{~ms}$ \\
$104-204$ & Skew & 0.9999 \\
\multirow{2}{*}{$204-200$} & Offset & $175 \mathrm{~ms}$ \\
& Skew & 1.0010 \\
$204-201$ & Offset & $136 \mathrm{~ms}$ \\
& Skew & 1.0000 \\
$204-202$ & Offset & $134 \mathrm{~ms}$ \\
& Skew & 0.9999 \\
$204-203$ & Offset & $163 \mathrm{~ms}$ \\
& Skew & 1.0030 \\
\hline
\end{tabular}

local clocks of all the nodes in the network to synchronize them with respect to the reference clock. Results of corrected clocks with respect to reference clock for all the nodes in the network at the end of cycle 2 have been tabulated in columns 4 and 8 for levela 1 and 2 in the network, respectively, in Table 4 .

As discussed in Section 4, new $\mathrm{CH}$ is elected every 5th cycle and takes effect as new $\mathrm{CH}$ two cycles after it has been elected. When network starts, initial $\mathrm{CH}$ nodes have been elected during 5 th cycle and these nodes take effect as new $\mathrm{CH}$ nodes in the network during 7 th cycle on network operation. In Table 5, we have represented skew and offset parameters obtained from cycles 8 and 9 in columns 3-4 and 7-8 for levels 1 and 2, respectively. Analysis of skew and offset parameters shows that clock synchronization works robustly even after $\mathrm{CH}$ rotation.

New nodes are dynamically added during hardware implementation of 3D airborne network during cycles 8 and 9 at levels 1 and 2, respectively. These newly added CN nodes take effect as $\mathrm{CH}$ nodes of their respective cluster during cycle 13 which is based on Cluster Head rotation policy. Hence, to reflect the effect of dynamic addition of nodes in the network, we have taken into account the values of skew and offset parameters obtained at the end of cycle 13 in Table 6 . Analyzing columns 3 and 6 from Table 6 and comparing the values obtained for skew and offset parameters at cycle 13 with cycle 2 when initial clock correction technique was applied, we can conclude that dynamic node addition at different levels in the network has no effect on skew and offset parameters of multihop Tri-Message time synchronization protocol.

5.2. Energy Consumption Analysis of the Network. When network starts, N100 and N200 are selected as Cluster Head
TABLE 7: Depleted energy during cycles 1, 2, and 5.

\begin{tabular}{lccc}
\hline Node & \multicolumn{3}{c}{$\begin{array}{c}\text { Depleted energy }(\mathrm{mJ}) \\
\text { Cycle 2 }\end{array}$} \\
\hline 100 & 12.8 & 12.85 & Cycle 5 \\
101 & 2.26 & 2.3 & 12.7 \\
102 & 2.2 & 2.22 & 2.18 \\
103 & 2.1 & 2.34 & 2.24 \\
200 & 10 & 10.1 & 2.28 \\
201 & 2.20 & 2.34 & 10 \\
202 & 2.42 & 2.26 & 2.26 \\
203 & 2.22 & 2.18 & 2.32 \\
\hline
\end{tabular}

TABLE 8: Depleted energy during cycles 8, 9, and 11.

\begin{tabular}{lccc}
\hline Node & \multicolumn{3}{c}{$\begin{array}{c}\text { Depleted energy }(\mathrm{mJ}) \\
\text { Cycle 9 }\end{array}$} \\
\hline 100 & 2.40 & 2.26 & Cycle 11 \\
101 & 15.4 & 15.43 & 2.38 \\
102 & 2.40 & 2.26 & 15.42 \\
103 & 2.30 & 2.26 & 2.38 \\
104 & 2.38 & 2.20 & 2.38 \\
200 & 2.20 & 2.34 & 2.34 \\
201 & 12.8 & 15.4 & 2.26 \\
202 & 2.26 & 2.3 & 15.43 \\
203 & 2.20 & 2.34 & 2.18 \\
204 & - & 2.34 & 2.26 \\
\hline
\end{tabular}

TABle 9: Depleted energy during cycle 13.

\begin{tabular}{lccc}
\hline Node & \multicolumn{3}{c}{$\begin{array}{c}\text { Depleted energy }(\mathrm{mJ}) \\
\text { Cycle 15 }\end{array}$} \\
\hline 100 & 2.40 & 2.30 & Cycle 17 \\
101 & 2.38 & 2.38 & 2.38 \\
102 & 2.3 & 2.34 & 2.34 \\
103 & 2.34 & 2.26 & 2.34 \\
104 & 15.3 & 15.4 & 2.18 \\
200 & 2.20 & 2.42 & 15.44 \\
201 & 2.18 & 2.26 & 2.22 \\
202 & 2.3 & 2.22 & 2.28 \\
203 & 2.26 & 2.2 & 2.34 \\
204 & 15.42 & 15.4 & 2.1 \\
\hline
\end{tabular}

nodes for the clusters formed at levels 1 and 2, respectively. Initially there are 4 nodes in each cluster which includes one Cluster Head node and 3 Cluster Nodes. Sample calculations for energy depletion during first cycle are shown below which can be verified from depleted energy values obtained during hardware implementation of the network in Table 7.

Tables 7, 8, and 9 represent depleted energy levels of all the nodes in the network.

Using (10), (11), and (12) and taking into account the different types of packets mentioned in Table 3 depleted energy for N100 during cycle 1 can be calculated easily. 
The values of $I_{T X_{P H}}, I_{T X_{P L}}$, and $I_{R X}$ have been taken from [34]. These values correspond to current consumption at transmission power levels 7 and 23, respectively

$$
\begin{aligned}
E_{\mathrm{N} 100} & =E_{\mathrm{TX}_{\mathrm{N} 100}}+E_{\mathrm{RX}_{\mathrm{N} 100}}, \\
E_{\mathrm{TX}_{\mathrm{N} 100}} & =4.0193 \mathrm{~mJ}, \\
E_{\mathrm{RX}_{\mathrm{N} 100}} & =8.8410 \mathrm{~mJ} .
\end{aligned}
$$

Hence,

$$
E_{\mathrm{N} 100}=E_{\mathrm{TX}_{\mathrm{N} 100}}+E_{\mathrm{RX}_{\mathrm{N} 100}}=12.86 \mathrm{~mJ} .
$$

Similarly depleted energy for N200 can be calculated as

$$
E_{\mathrm{N} 200}=3.0390 \mathrm{~mJ}+7.0207 \mathrm{~mJ}=10.0597 \mathrm{~mJ} .
$$

Depleted energy of any Cluster Node in the network can be calculated as

$$
E_{\mathrm{CN}}=0.8939 \mathrm{~mJ}+1.371 \mathrm{~mJ}=2.265 \mathrm{~mJ} .
$$

In fifth cycle, Nodes N101 and N201 are elected as CH nodes in level 1 and level 2 , respectively. They take effect as new $\mathrm{CH}$ nodes in their respective levels during seventh cycle. In Table 8 depleted energies of nodes during cycle 8,9 , and 11 have been tabulated.

New nodes have been added during 8th and 9th cycle in level 1 and level 2, respectively. In cycle 11, these newly added nodes have been elected as new $\mathrm{CH}$ nodes for their respective levels. These nodes take effect from cycle 13 onward. The depleted energies of these nodes can be verified from Table 9 .

5.3. Cluster Head Rotation. As discussed in Section 4.2, Cluster Head rotation policy has been utilized during hardware implementation of 3D airborne network. Every fifth cycle, $\mathrm{CN}$ nodes send their depleted energy values to their respective $\mathrm{CH}$ nodes along with the sensed data. $\mathrm{CH}$ nodes elect new $\mathrm{CH}$ for their levels based upon the node which has depleted minimum energy from their respective clusters. If two or more nodes are found to have depleted same amount of energy, node with lower node id is elected. Figure 7 shows graph of depleted energies of N100, N101, and N102 from level 1 over first 10 cycles of network operation.

During first five cycles of network operation, N100 acts as $\mathrm{CH}$ for level 1. From Section 5.2, it depletes $12.86 \mathrm{~mJ}$ energy in every cycle. In this graph, we have also included depleted energy values for Nodes N101 and N102. Since both of them act as $\mathrm{CN}$ nodes during first five cycles, they consume $2.265 \mathrm{~mJ}$ energy in every cycle. N101 and N102 consume the same amount of energy and when new $\mathrm{CH}$ has to be elected during fifth cycle; N101 has been given precedence over N102 because of its lower node id. In cycle 7, N101 becomes new $\mathrm{CH}$ for level 1 and it depletes $12.86 \mathrm{~mJ}$ energy during rest of the cycles until next $\mathrm{CH}$ has been elected.

5.4. Packet Delivery Ratio (PDR). In the hardware platform, repetitive master cycles of DCB-TDMA MAC have been implemented. In the control cycle phase of these master cycle,

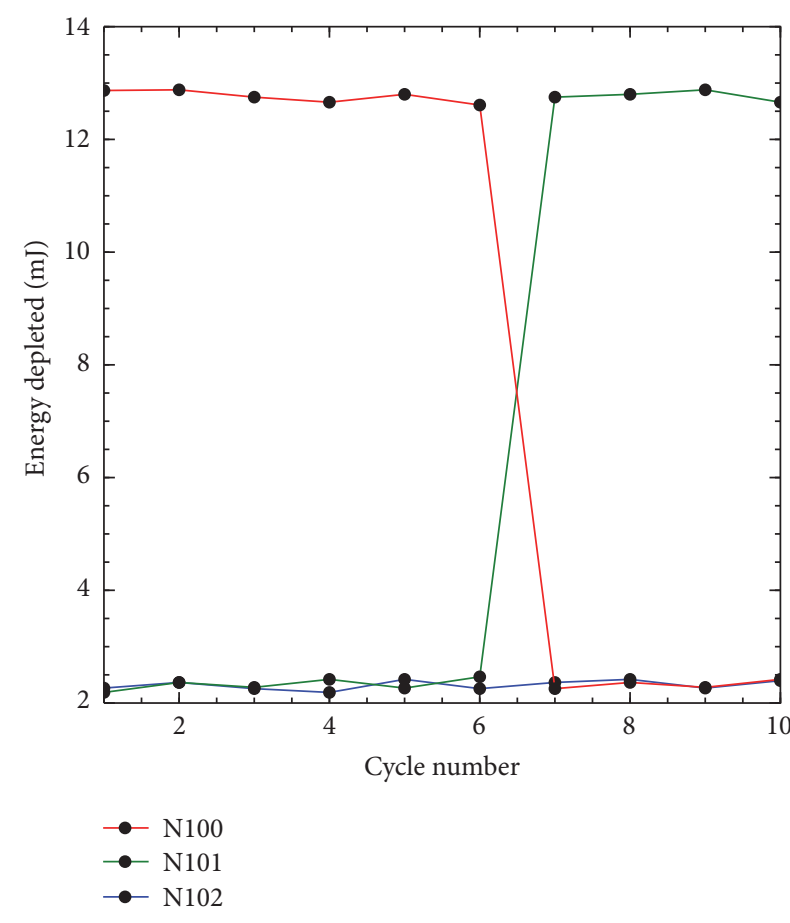

FIgURE 7: Cluster Head rotation policy.

time synchronization has been conducted whereas in the data cycle phase, sensed data has been collected on the ground based Base Station node. After collection of data at BS, sleep cycle (of equal duration of wake cycle) has been scheduled. During the repetition of master cycles it has been observed that all packets have been delivered successfully to intended receiver node, giving the PDR as $100 \%$.

In Section 6, conclusions of this paper have been provided.

\section{Conclusions}

In this paper, network architecture of 3D airborne network has been proposed. This network architecture is cluster-based hierarchical network consisting of static nodes deployed at varying heights above ground level. For this network architecture, a protocol stack consisting of TDMA based MAC protocol, dynamic routing, time synchronization, Cluster Head rotation, and power level management has been proposed. This complete stack has been deployed on the 3D network using TelosB nodes. This 3D airborne network architecture can be used to monitor quality of the air. Air pollutants are monitored to derive AQI for an area. It has been demonstrated in the paper that this proposed framework is energy efficient and hence suitable for longevity of the deployed network. Energy efficient network operation has been showcased using following features:

(1) The network nodes follow a sleep-wake cycle of $50 \%$. Along with this, since a TDMA based MAC protocol has been utilized, nodes sleep for duration other than preallotted time slots conserving energy. 
(2) Energy balancing among network nodes is possible because of Cluster Head rotation, which is performed in regular intervals. It also prevents the network from complete failure in backbone link.

(3) Multihop communication between $\mathrm{CH}$ nodes and BS node consumes less power compared to LEACH clustering protocol translated for 3D WSNs. Also Cluster Head rotation policy helps in increasing the network lifespan compared to clustering protocol proposed in [33].

(4) An energy efficient (lightweight) time synchronization protocol has been utilized on the network. This time synchronization protocol requires least amount of message exchanges to achieve time synchronization and hence consumes lesser power, memory, and time in a process.

(5) For communication at various ranges, optimum power levels of transmission have been utilized. Nodes dynamically switch the power levels based on the range of communication optimizing the battery usage.

\section{Competing Interests}

The authors declare that there is no conflict of interests regarding the publication of this paper.

\section{References}

[1] J. Yick, B. Mukherjee, and D. Ghosal, "Wireless sensor network survey," Computer Networks, vol. 52, no. 12, pp. 2292-2330, 2008.

[2] J. A. Stankovic, "Wireless sensor networks," Computer, vol. 41, no. 10, pp. 92-95, 2008.

[3] M. P. Đurišić, Z. Tafa, G. Dimić, and V. Milutinović, "A survey of military applications of wireless sensor networks," in Proceedings of the 1st Mediterranean Conference on Embedded Computing (MECO '12), pp. 196-199, IEEE, Bar, Montenegro, June 2012.

[4] A. Mainwaring, D. Culler, J. Polastre, R. Szewczyk, and J. Anderson, "Wireless sensor networks for habitat monitoring," in Proceedings of the 1st ACM International Workshop on Wireless Sensor Networks and Applications, pp. 88-97, Atlanta, Ga, USA, September 2002.

[5] X. Shen, Z. Wang, and Y. Sun, "Wireless sensor networks for industrial applications," in Proceedings of the Fifth World Congress on Intelligent Control and Automation (WCICA '04), pp. 3636-3640, June 2004.

[6] J. Allred, A. B. Hasan, S. Panichsakul et al., "SensorFlock: an airborne wireless sensor network of micro-air vehicles," in Proceedings of the 5th ACM International Conference on Embedded Networked Sensor Systems (SenSys '07), pp. 117-129, ACM, Sydney, Australia, November 2007.

[7] M. Erol-Kantarci, H. T. Mouftah, and S. Oktug, "A survey of architectures and localization techniques for underwater acoustic sensor networks," IEEE Communications Surveys \& Tutorials, vol. 13, no. 3, pp. 487-502, 2011.

[8] E. Kulla, S. Sakamoto, M. Ikeda, and L. Barolli, "MANET approaches for airborne networks: a survey," in Proceedings of the 16th International Conference on Network-Based Information
Systems (NBiS '13), pp. 66-70, Gwangju, South Korea, September 2013.

[9] I. F. Akyildiz, D. Pompili, and T. Melodia, "Underwater acoustic sensor networks: research challenges," Ad Hoc Networks, vol. 3, no. 3, pp. 257-279, 2005.

[10] World Health Organization, Air Quality Guidelines: Global Update 2005: Particulate Matter, Ozone, Nitrogen Dioxide, and Sulfur Dioxide, World Health Organization, 2006.

[11] A. Joshi, S. Dhongdi, K. Anupama, P. Nahar, and R. Sethunathan, "Implementation of protocol stack for threedimensional wireless sensor network," Procedia Computer Science, vol. 89, pp. 193-202, 2016.

[12] C. Tian, H. Jiang, X. Liu, X. Wang, W. Liu, and Y. Wang, "Trimessage: a lightweight time synchronization protocol for high latency and resource-constrained networks," in Proceedings of the IEEE International Conference on Communications (ICC '09), pp. 1-5, IEEE, Dresden, Germany, June 2009.

[13] Y.-C. Wu, Q. Chaudhari, and E. Serpedin, "Clock synchronization of wireless sensor networks," IEEE Signal Processing Magazine, vol. 28, no. 1, pp. 124-138, 2011.

[14] A. Nasridinov, S.-Y. Ihm, Y.-S. Jeong, and Y.-H. Park, "Event detection in wireless sensor networks: survey and challenges," in Mobile, Ubiquitous, and Intelligent Computing, vol. 274 of Lecture Notes in Electrical Engineering, pp. 585-590, Springer, Berlin, Germany, 2014.

[15] M. T. Lazarescu, "Design of a WSN platform for long-term environmental monitoring for IoT applications," IEEE Journal on Emerging and Selected Topics in Circuits and Systems, vol. 3, no. 1, pp. 45-54, 2013.

[16] É. L. Souza, E. F. Nakamura, and R. W. Pazzi, “Target tracking for sensor networks: a survey," ACM Computing Surveys (CSUR), vol. 49, no. 2, article 30, 2016.

[17] G. Lu, N. Sadagopan, B. Krishnamachari, and A. Goel, "Delay efficient sleep scheduling in wireless sensor networks," in Proceedings of the 24th Annual Joint Conference of the IEEE Computer and Communications Societies., pp. 2470-2481, Miami, Fla, USA.

[18] D. L. Mills, "Internet time synchronization: the network time protocol," IEEE Transactions on Communications, vol. 39, no. 10, pp. 1482-1493, 1991.

[19] J. Elson and K. Römer, "Wireless sensor networks: a new regime for time synchronization," ACM SIGCOMM Computer Communication Review, vol. 33, no. 1, pp. 149-154, 2003.

[20] S. Ganeriwal, R. Kumar, and M. B. Srivastava, "Timing-sync protocol for sensor networks," in Proceedings of the 1st International Conference on Embedded Networked Sensor Systems, pp. 138-149, ACM, 2003.

[21] M. Maróti, B. Kusy, G. Simon, and Á. Lédeczi, “The flooding time synchronization protocol," in Proceedings of the 2nd International Conference on Embedded Networked Sensor Systems, pp. 39-49, ACM, 2004.

[22] K.-L. Noh, Y.-C. Wu, K. Qaraqe, and B. W. Suter, "Extension of pairwise broadcast clock synchronization for multicluster sensor networks," Eurasip Journal on Advances in Signal Processing, vol. 2008, Article ID 286168, 2008.

[23] P. Suriyachai, U. Roedig, and A. Scott, "A survey of MAC protocols for mission-critical applications in wireless sensor networks," IEEE Communications Surveys \& Tutorials, vol. 14, no. 2, pp. 240-264, 2012.

[24] W. Ye, J. Heidemann, and D. Estrin, "An energy-efficient mac protocol for wireless sensor networks," in Proceedings of 
the 21st Annual Joint Conference of the IEEE Computer and Communications Societies (INFOCOM '02), vol. 3, pp. 15671576, IEEE, 2002.

[25] M. I. Brownfield, K. Mehrjoo, A. S. Fayez, and N. J. Davis, "Wireless sensor network energy-adaptive mac protocol," in Proceedings of the 3rd IEEE Consumer Communications and Networking Conference (CCNC '06), January 2006.

[26] U. A. Patil, S. V. Modi, and B. J. Suma, "A survey: MAC layer protocol for wireless sensor networks," International Journal of Emerging Technology and Advanced Engineering, vol. 3, no. 9, pp. 203-211, 2013.

[27] V. Rajendran, K. Obraczka, and J. J. Garcia-Luna-Aceves, "Energy-efficient, collision-free medium access control for wireless sensor networks," Wireless Networks, vol. 12, no. 1, pp. 63-78, 2006.

[28] L. F. W. Van Hoesel, T. Nieberg, H. J. Kip, and P. J. M. Havinga, "Advantages of a TDMA based, energy-efficient, self-organizing MAC protocol for WSNs," in Proceedings of the IEEE 59th Vehicular Technology Conference (VTC '04-Spring): Towards a Global Wireless World, vol. 3, pp. 1598-1602, IEEE, Milan, Italy, May 2004.

[29] M. Salajegheh, H. Soroush, and A. Kalis, "HYMAC: hybrid TDMA/FDMA medium access control protocol for wireless sensor networks," in Proceedings of the IEEE 18th International Symposium on Personal, Indoor and Mobile Radio Communications (PIMRC '07), pp. 1-5, IEEE, Athens, Greece, September 2007.

[30] K. Benkic, "Proposed use of a CDMA technique in wireless sensor networks," in Proceedings of the 6th EURASIP Conference Focused on Speech and Image Processing, Multimedia Communications and Services, 14th International Workshop on Systems, Signals and Image Processing, pp. 343-348, IEEE, 2007.

[31] M. J. Handy, M. Haase, and D. Timmermann, "Low energy adaptive clustering hierarchy with deterministic cluster-head selection," in Proceedings of the 4th International Workshop on Mobile and Wireless Communications Network (MWCN'02), pp. 368-372, IEEE, Stockholm, Sweden, September 2002.

[32] M. Baghouri, A. Hajraoui, and S. Chakkor, "Low energy adaptive clustering hierarchy for three-dimensional wireless sensor network," in Recent Advances in Communications, pp. 214-218, 2015.

[33] N. Attarzadeh and M. Mehrani, "A new three dimensional clustering method for wireless sensor networks," Global Journal of Computer Science and Technology, vol. 11, no. 6, pp. 54-60, 2011.

[34] J. Lee and K. Chung, "An efficient transmission power control scheme for temperature variation in wireless sensor networks," Sensors, vol. 11, no. 3, pp. 3078-3093, 2011. 


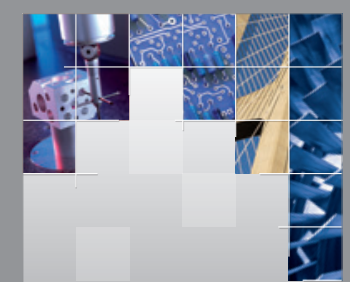

\section{Enfincering}
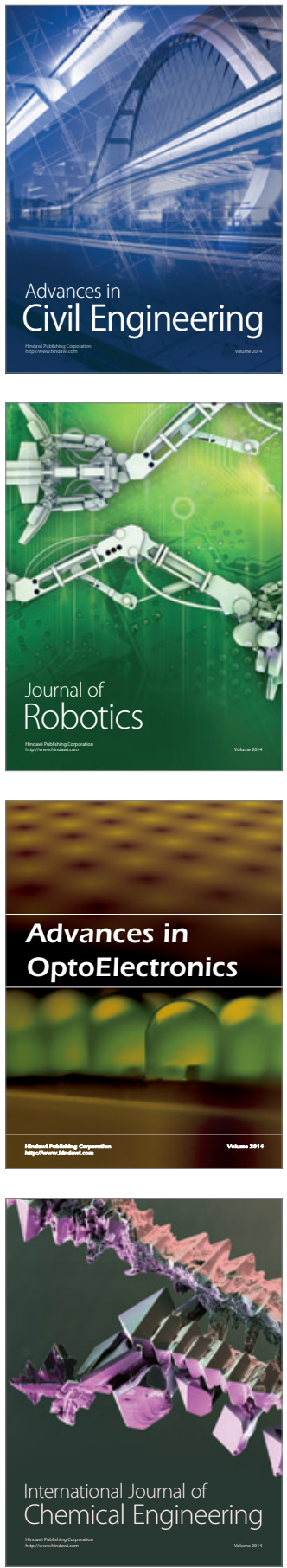

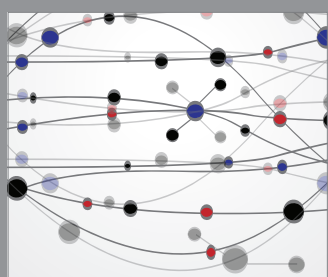

The Scientific World Journal

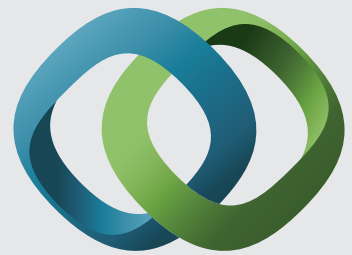

\section{Hindawi}

Submit your manuscripts at

https://www.hindawi.com
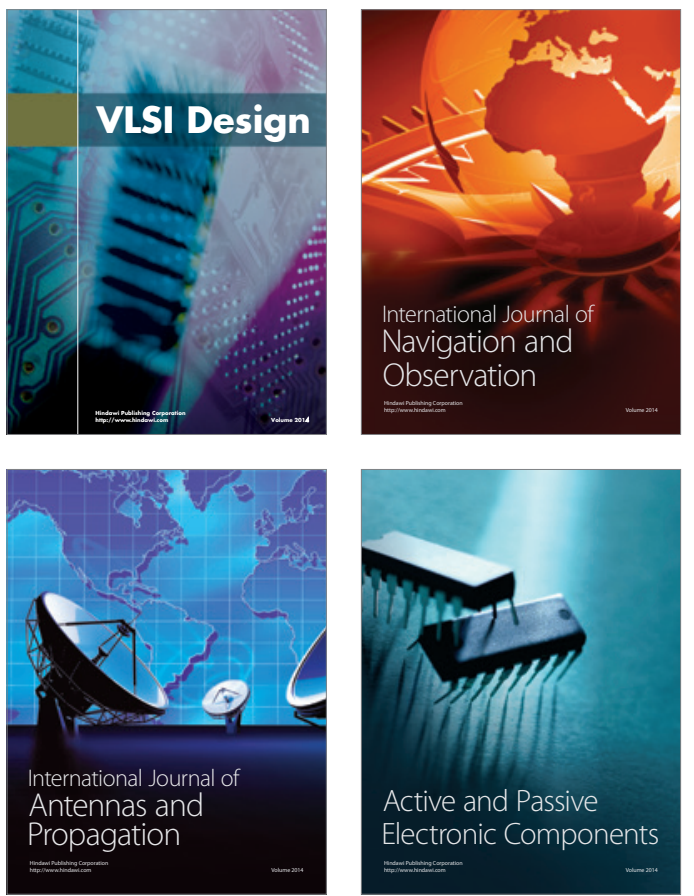
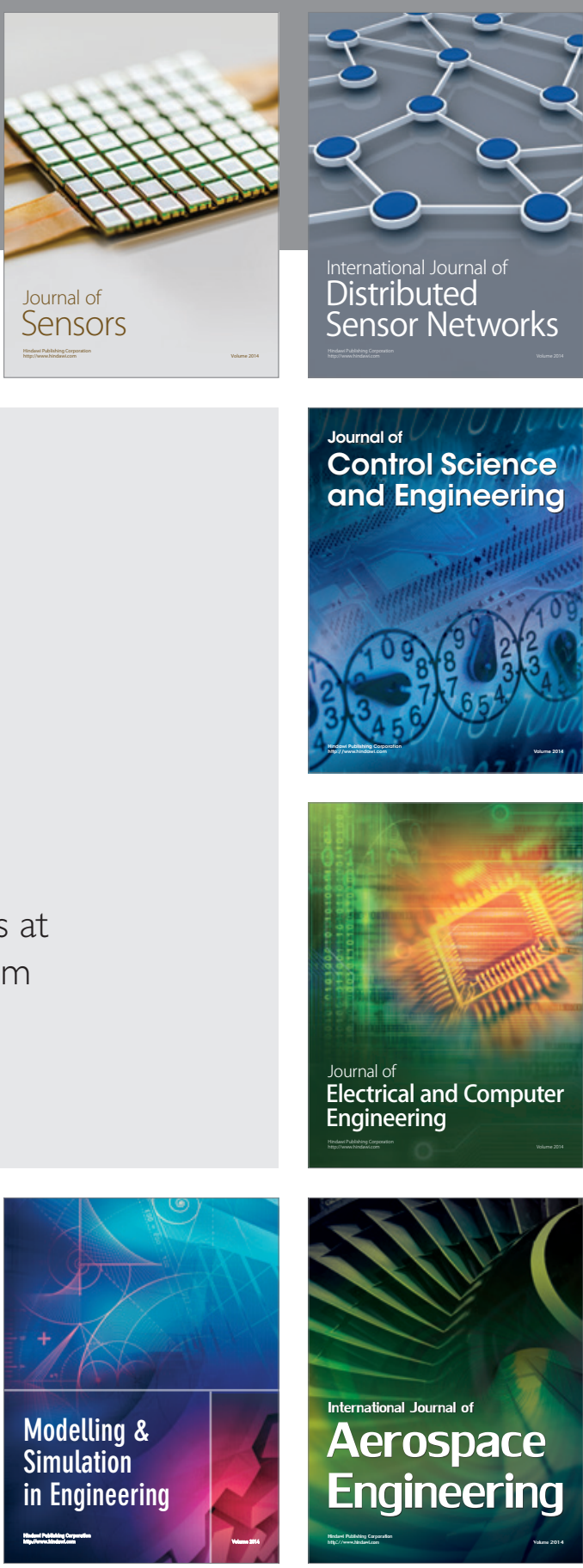

International Journal of

Distributed

Sensor Networks

$-$

Joumal of

Control Science

and Engineering
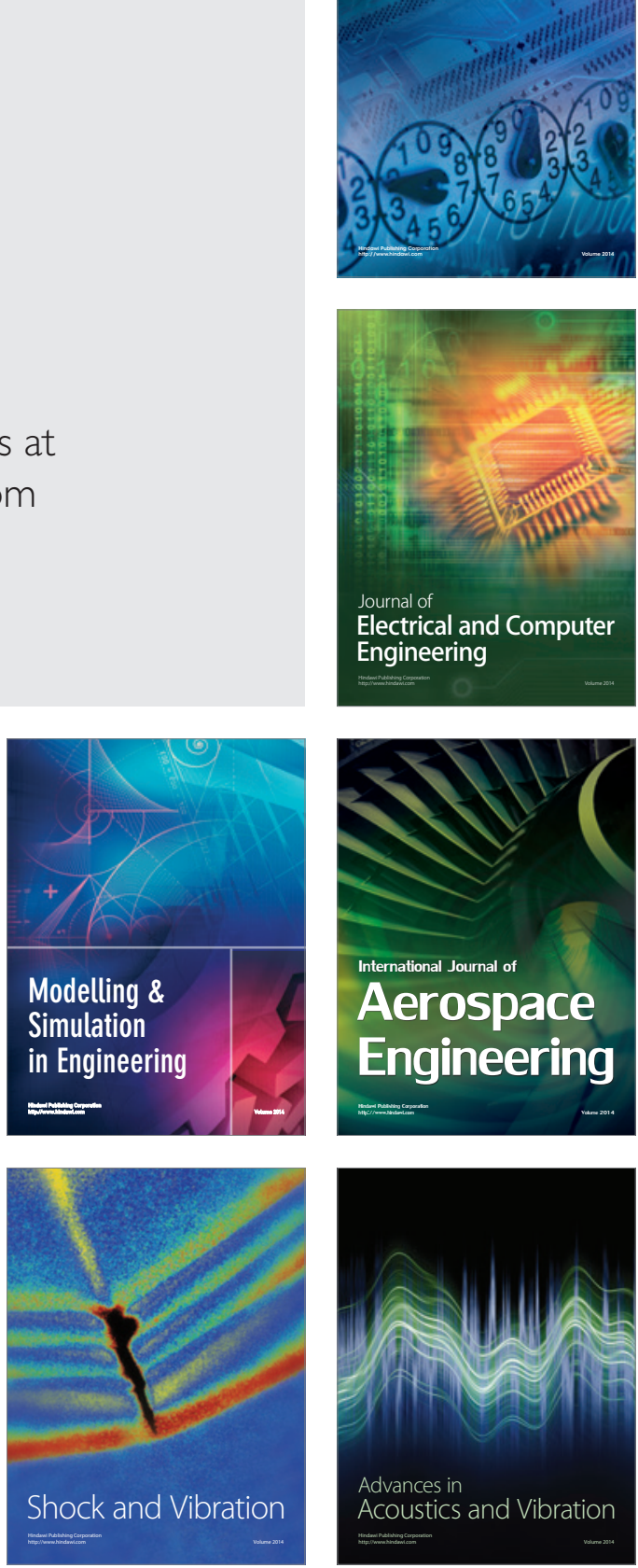